\title{
DINÁMICA SOCIO-PRODUCTIVA Y COMERCIALIZACIÓN EN LAS FERIAS SOLIDARIAS DE LA CIUDAD DE IBARRA
}

\section{SOCIO-PRODUCTIVE DYNAMICS AND COMMERCIALIZATION ON SOLIDARY FAIRS AT THE IBARRA CITY}

\begin{abstract}
Juan Carlos López Gutiérrez, Ph.D.
Doctor en Ciencias de la Educación (Cuba). Docente-Investigador de la Facultad de Educación, Ciencia y Tecnología de la Universidad Técnica del Norte, Ecuador. iclopez@utn.edu.ec

Pedro Segundo Quelal Onofre, Mgs. Magíster en Desarrollo Regional y Planificación Territorial (Ecuador). Docente-Investigador de la Facultad de Educación, Ciencia y Tecnología de la Universidad Técnica del Norte, Ecuador. spquelal@utn.edu.ec José Daniel Rosillo Solano, Mgs. Magíster en Gerencia Educativa (Ecuador). Docente de la Universidad Estatal de Bolívar, Ecuador. jdrosillo81@gmail.com
\end{abstract}

\section{ARTÍCULO DE INVESTIGACIÓN}

Recibido: 30 de noviembre de 2018.

Aceptado: 22 de febrero de 2019.

\section{RESUMEN}

Las ferias solidarias constituyen un sistema de comercialización directo - consumidor. Su dinámica más interesante es que el mismo productor se encarga de asignarle un valor agregado a su producto para ser ofertado con mayor oportunidad. El objetivo del artículo es describir los volúmenes de venta, derivados de la comercialización en las ferias solidarias de la ciudad de Ibarra, como resultado de su dinámica socio - productiva. Se consideran 
los indicadores: sistema de comercialización y volumen de ventas. El enfoque metodológico asumido en la investigación fue el método mixto. Los resultados de la investigación muestran que las dinámicas de comercialización en las ferias presentes en lbarra, expresan relaciones sociales e interculturales. El volumen total de ventas ha aumentado permitiendo que los ingresos generados contribuyan a sustentar a los productores - feriantes, sus familias y otros sectores de la provincia.

Palabras clave: Dinámica socio - productiva, ferias solidarias, sistema de comercialización.

\section{ABSTRACT}

The solidary fair constitutes a direct consumer marketing system. It most interesting dynamic is that producers are responsible for adding value to their product in order to get a major market opportunity. The objective of the paper is to describe the sales volumes derived from the solidary fairs at the Ibarra City, as result of its socio-productive dynamics. The marketing system and the sales volumes were considered as indicators. The mixed method was used as research design. Also, documentary analysis and semi-structured interview were used as specific research techniques. The result shows in what way the dynamics of commercialization in the solidary fairs, express social and intercultural relations. Total sales volumes are being increased, made the incomes contribute to sustain the producers-fair people and their families and other sectors of the province too.

Keywords: Solidary fairs, socio - productive dynamics, marketing system.

\section{INTRODUCCIÓN}

El presente trabajo se ha apoyado en la tesis sobre "la economía solidaria, que sustenta la propuesta de ferias solidarias, representa un paradigma alternativo de desarrollo" (Murillo \& Lacroix, 2014, p.102) el cual se construye articulando la experiencia de las organizaciones, sus prácticas y costumbre a la dinámica económica que realizan. Las ferias de "productos agroalimentarios, las que valorizan productos alimenticios locales, referenciados cultural y territorialmente" (Pierri \& Valiente, 2010, p.10). Las mismas se vinculan a los temas alimentarios, asumiendo cualesquiera de las clasificaciones: libres o 
solidarias, así "como forma alternativa" según la Comisión Económica para América Latina y el Caribe (CEPAL, 2016, p.104). Estas son "espacios de intercambio, comercialización, aprendizaje y articulación" (Murillo \& Lacroix, 2014, p.102).

La economía social y solidaria ubica en el centro del proceso a las personas y su trabajo, aspectos que permiten la mejora de sus capacidades de iniciativa y creatividad. A su vez se fomenta el aprendizaje y el trabajo cooperativo entre personas y organizaciones en los diferentes ámbitos de la localidad, la región o el país. Ello permite comprender la participación de estas últimas en el desarrollo local sostenible y comunitario a través de su implicación en redes dentro del mismo ámbito geográfico y en otros más amplios (Guridi \& Pérez de Mendiguren, 2008).

Las ferias "son tan antiguas como las ciudades y la diferenciación del trabajo en la sociedad. Surgen por la necesidad de intercambiar bienes producidos en distintas localidades (CEPAL, 2016, p.104). Estas constituyen formas más actuales de comercialización de productos, ya sea en espacios al aire libre o cerrados, en la que confluyen productores feriantes ${ }^{1}$ y consumidores. Pierri y Valiente (2010) dicen que estas "son eventos periódicos, que ocurren en espacios públicos, donde hombres y mujeres realizan intercambios de mercancías, con la finalidad de garantizar sus condiciones materiales de vida. Son parte de circuitos locales de comercialización" (p.11).

Estas ferias, se han ido estableciendo como espacios alternativos de respuestas ante modelos neoliberales y excluyentes de sectores de la sociedad, entre los cuales se encuentran aquellos ligados a la agricultura familiar.

En un nivel más amplio y como lo mencionan algunos estudios alrededor de las ferias campesinas, estas "son experimentos concretos de una propuesta de economía solidaria, articulada a nivel regional o a nivel de país por redes como el Movimiento de Economía Social y Solidaria del Ecuador o el Colectivo Agroecológico" (Murillo \& Lacroix, 2014, p.103). Para Gago, Cielo \& Gachet (2018) estas se consideran como espacios de experimentación

${ }^{1}$ Una denominación que los autores han preferido asumir es la de productores - feriantes, considerando las denominaciones que se han identificado (solidarias, libres), en la cual los comerciantes van a la feria con productos cosechados por ellos, de producción propia o en su defecto, en un mayor por ciento de producción propia. 
según las condiciones de cada región, incluyendo aspectos referidos a formas productivas, de comercialización, de construcción de vínculos que expresan la creatividad y la capacidad de innovación popular.

En la literatura se han abordado estudios sobre las ferias libres o solidarias en la provincia de Imbabura (Mera, 2013; Calle y Zapatta, 2013; Murillo y Lacroix, 2014; Zárate, 2015; Pinto, 2016; Guevara, Arciniegas \& Guerrero, 2017). Sin embargo, un análisis que considere la dinámica socio - productiva, concentrándose en los circuitos de comercialización y ventas en la ciudad de Ibarra no ha sido explicitado.

Por medio de la dinámica es posible dar cuenta de los logros y posibilidades sociales para el mejoramiento de la calidad de vida humana. En este sentido, lo que se denomina dinamismo de la sociedad y de la historia se convierten en conceptos inter disciplinarios, porque al estudiar las actividades humanas a través del tiempo, lo que realmente se desea conocer es el desarrollo de la sociedad en la economía, la política, la religión, las ideologías, el arte, etc., todo en su conjunto (Álvarez \& Maldonado, 2009).

Una dinámica socio - productiva en el contexto de las ferias pudiera considerar al conjunto de los movimientos, cambios e interactividad de todos los miembros o socios de estas. Ello sintetiza las diversas disciplinas o ciencias sociales fundamentalmente para explicar el hecho, como la sociología y la economía. Así se caracteriza la dinámica establecida en las ferias a partir de sus relaciones con los circuitos de comercialización y las ventas que realizan. La interrelación de éstos dos elementos constituye la base de la dinámica socio productiva. La interrelación planteada permite comprender el desarrollo de las ferias como parte de un ecosistema social que se evidencia en la ciudad de lbarra.

El objetivo del estudio consistió en describir los volúmenes de venta, derivados de la comercialización en las ferias solidarias de la ciudad de lbarra, como resultado de su dinámica socio - productiva.

\section{MATERIALES Y MÉTODOS}


Desde el punto de vista metodológico, el presente trabajo se encuadra dentro de lo que se conoce como método mixto, el cual asume enfoques cualitativos y cuantitativos (Pérez, 2011). El diseño del método mixto permite integrar estrategias y procedimientos de otro enfoque de investigación para estudiar un mismo objeto (Bolívar, 2008). Consiste en un único estudio que utiliza estrategias múltiples o mixtas para responder a las preguntas de investigación y/o comprobar hipótesis (Driessnack, Sousa y Costa, 2007). En la presente investigación se utilizó la tipología de investigación confirmatoria, con datos cuantitativos y análisis cualitativo (Pérez, 2011).

La muestra seleccionada tuvo un carácter intencional (Marsollier, 2018; Vega \& Ramírez, 2018; Claveria \& Carrasco, 2018) focalizada en cuatro ferias solidarias de la ciudad de Ibarra de la provincia de Imbabura, conformada por 199 productores - feriantes. De ellos el $84,9 \%$ de género femenino y el $15,1 \%$ masculino. Todos poseen como característica para su selección que son comerciantes y van a la feria con productos cosechados por ellos, de producción propia o en su defecto, en un mayor por ciento de producción propia.

El diseño mixto utilizado contempló dos fases La fase I respondió a un enfoque cuantitativo, utilizando el método descriptivo (Abreu, 2014). Se asumió como premisa que los productores - feriantes poseen los datos necesarios que contribuyen a la argumentación de una dinámica socio - productiva, pues son ellos los que la construyen. Se empleó un cuestionario semiestructurado para recopilar información. La fase II se apoyó en el método analítico. Su aplicación permitió enfatizar en la dinámica socio - productiva de los actores que hacen la feria, en sus aspectos cognitivos, afectivos y contextuales, lo que acerca al conocimiento de un objeto de estudio.

\section{RESULTADOS Y DISCUSIÓN}

\section{Contexto general de Ibarra}

La ciudad de San Miguel de Ibarra está situada al norte del Ecuador en la provincia de Imbabura. 
El cantón lbarra es reconocido por ser multicultural esto en gran medida al considerar un cantón multiétnico. Según datos del Instituto Nacional de Estadística y Censos (INEC, 2010) el 16\% de la población de Ibarra se auto identifica como Indígena, el $8.7 \%$ como afroecuatoriana y $0.3 \%$ como montubia. El restante $78 \%$ se auto identifica como mestiza y otra. El cantón Ibarra es el cantón imbabureño que más población afroecuatoriana concentra en relación a otros cantones pues un 74\% de la población afro imbabureña vive en Ibarra (Velarde, 2013, p.27-28).

¿Qué nivel de representatividad presentan las etnias presentes en el cantón lbarra con relación a los productores - feriantes en las ferias solidarias?

La muestra con la que se trabajó, el 61,8\% se autodefinen de etnia mestizos, el 36,7\% como indígenas y solo el 1,5\% como afroecuatorianos.

Tabla 1. Comparación de grupos étnicos en el cantón lbarra y productores - feriantes

\begin{tabular}{lcc}
\hline \multicolumn{1}{c}{ Grupo étnico } & (INEC, 2010; Velarde, 2013) & Productores - Feriantes \\
\hline Blanco & $\star 2$ & $0 \%$ \\
Mestiza + mulato & $78 \%{ }^{* 3}$ & $61.8 \%$ \\
Indígena & $16 \%$ & $36.7 \%$ \\
Afroecuatoriano & $4.93 \%$ & $1.5 \%$ \\
\hline
\end{tabular}

Fuente: Elaboración propia a partir del INEC (2010) y Velarde (2013).

Aun y cuando los mestizos son más representativos del cantón, los indígenas tienen una mayor presencia en las ferias (36.7\%). Ello es compatible con la multiculturalidad en gran medida por ser un cantón multiétnico (Velarde, 2013). Respecto a la etnia Afroecuatoriana, la representatividad como productores - feriantes es solo $1.5 \%$, lo que significa 41 veces menos si lo comparamos con la presencia mestiza y 24.3 veces menos comparado con la presencia indígena.

Otro aspecto del cantón lbarra es el relativo a la estructura económica. Según los datos reportados por el INEC (2010) y Velarde (2013) el sector agropecuario es el que menor

\footnotetext{
* No reportan datos.

* Se incluye una subdivisión de etnia mulatos.
} 
representatividad posee con el 11,61\%, lo que lo ubica en desventaja con relación a los otros dos sectores predominantes.

Tabla 2. Estructura económica Cantón Ibarra

\begin{tabular}{|c|c|c|}
\hline $\begin{array}{c}\text { Sectores } \\
\text { productivos }\end{array}$ & $\%$ que representa & Principales actividades \\
\hline Servicios & $\begin{array}{l}\text { - Representa } \\
58,02 \% \text {. }\end{array}$ & $\begin{array}{l}\text { el Comercio al por mayor y menor, } \\
\text { enseñanza, administración } \\
\text { pública y transporte. }\end{array}$ \\
\hline Industrias & $\begin{array}{l}\text { - Representa } \\
\text { 19,38\%. }\end{array}$ & $\begin{array}{l}\text { - Principales actividades: industria } \\
\text { manufacturera }\end{array}$ \\
\hline Agropecuario & $\begin{array}{l}\text { - Representa } \\
11,61 \% \text {. }\end{array}$ & $\begin{array}{l}\text { el Principales actividades agrícolas, } \\
\text { ganaderas, silvicultura y pesca }\end{array}$ \\
\hline
\end{tabular}

Fuente: Elaboración propia a partir del INEC (2010) y Velarde (2013).

\section{Sistema de comercialización}

Los resultados obtenidos permiten responder la interrogante: ¿constituyen las ferias solidarias de la ciudad de lbarra circuitos cortos de comercialización? Es importante identificar este aspecto pues contribuye a desarrollar relaciones de intercambio justo para los productores - feriantes y los consumidores, además de lograr establecer el desarrollo del sector agropecuario y el sustento económico para la agricultura familiar. Por otra parte, constituye el tercer sector productivo en importancia en el cantón. Este aspecto se relaciona con la estrategia política desarrollada por el estado ecuatoriano de ubicar en primer plano al ciudadano respecto al mercado y al capital, a partir de que "el Ministerio de Agricultura diseñó en el año 2007 un componente de redes comerciales que impulsa la estrategia para la comercialización de productos provenientes de organizaciones campesinas en circuitos cortos, cuyo propósito es generar la compra y venta directa del productor al consumidor" (Guevara et al., 2017, p.550).

Para estos autores, en la provincia de Imbabura, esta actividad se desarrolla desde el 2007. "El objetivo fue permitir el intercambio directo entre el productor y el consumidor, evitando 
el intermediario, con el fin de lograr precios más justos para los dos sectores y dinamizar la economía local fortaleciendo el sector agrícola (Guevara et al., 2017, p.551).

Una variable que contribuye al esclarecimiento de esta interrogante tiene que ver con el origen de los productos agroalimentarios comercializados. Este se convierte en un aspecto fundamental que permite determinar la incidencia de las ferias en un sistema de comercialización determinado. Los resultados expresan que la muestran estudiada se caracteriza por pertenecer a un sistema de comercialización directo - consumidor. En este el productor se vincula de forma directa al consumidor final, sin depender de algún otro agente directo para su comercialización. Su primer agente de transacción es el consumidor final. La dinámica más interesante en este sistema es que el mismo productor es el encargado de darle un valor agregado a su producto para ser ofertado con mayor oportunidad a este agente.

Tabla 3. Origen de los productos agroalimentarios

\begin{tabular}{lccc}
\hline $\begin{array}{c}\text { Origen de los } \\
\text { productos }\end{array}$ & Natural & $\begin{array}{c}\text { Tipo de producto } \\
\text { Semiprocesado }\end{array}$ & Procesado \\
agroalimentarios & & & $80,6 \%$ \\
\hline Propio & $75,9 \%$ & $81,4 \%$ & $3,2 \%$ \\
\hline
\end{tabular}

Fuente: Elaboración propia a partir de los datos recolectados.

Es importante resaltar que "en muchas de las ferias tradicionales, están presentes entre los vendedores tanto productores como comerciantes, sin que haya una distinción entre estas dos categorías para el consumidor" (CEPAL, 2016, p.104). Sin embargo, las ferias de venta directa, considerando la concepción del productor - feriante, donde los productos agroalimentarios son cosechados por ellos, son altamente consideradas por los consumidores, ante una postura responsable del consumo. Esta constituye la particularidad esencial para considerarlas como parte de los circuitos cortos de comercialización, los cuales reducen la distancia geográfica desde el origen de la producción agroalimentaria y el espacio de su comercialización. 
Los circuitos cortos de comercialización se caracterizan por precios más justos para el productor y el consumidor, una oferta productiva ecológica o más saludable, una mayor diversidad de los productos locales con énfasis en las variedades y razas nativas (Lacroix \& Cheng, 2014, p.14).

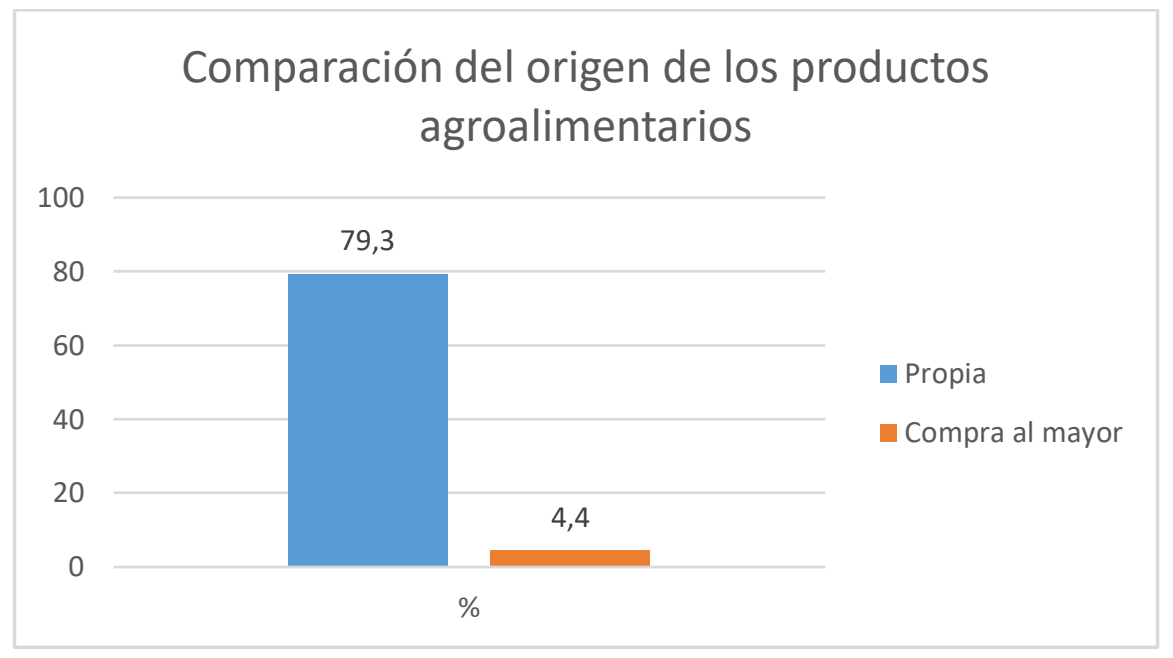

Gráfico 1. Comparación del origen de los productos agroalimentarios.

Fuente: Elaboración propia a partir de los datos recolectados.

Los resultados expresan un promedio del 79,3\% de origen propio de los productos agroalimentarios, lo que representa 18 veces más que los que han sido comprados al mayor $(4,4 \%)$. En respuesta a la interrogante planteada, las ferias solidarias de la ciudad de Ibarra si constituyen circuitos cortos de comercialización.

El auge de los circuitos cortos se debe fundamentalmente a una creciente demanda por parte de los consumidores, quienes buscan productos locales, auténticos, sanos y de temporada. Los productores, en tanto, buscan capturar un mayor valor en la producción, realizar un ahorro en otros segmentos de la cadena (CEPAL, 2016, p.13).

Estos resultados deben verse en comparación con la forma de articulación de los productores - feriantes de la provincia de Imbabura en general con cada uno de los subsistemas de comercialización (indirecto corto, indirecto largo y directo consumidor). Calle y Zapata (2013) reconocen que los mecanismos más frecuentes de comercialización corresponden al subsistema indirecto largo (50\%). Tal es la conclusión a la que se puede 
llegar cuando se observa la frecuencia con la que los distintos tipos de productores analizados se relacionan con cada uno de los subsistemas identificados.

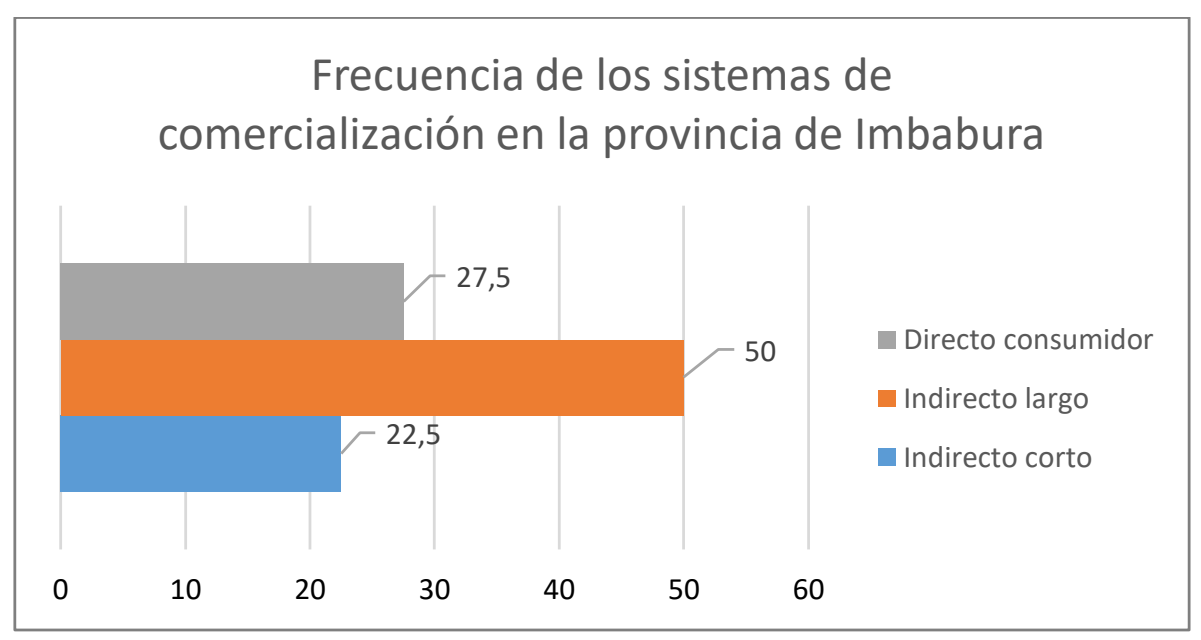

Gráfico 2. Frecuencia de los sistemas de comercialización en la provincia de Imbabura. Fuente: Elaboración propia a partir de Calle y Zapata (2013).

En el subsistema indirecto largo se identifica como principal agente de comercialización a los acopiadores transportistas que crean lazos de dependencia desde los productores, por las facilidades de créditos, entrega de insumos para los cultivos y de víveres de primera necesidad en la unidad productiva, entre otras. Estos aspectos les garantiza que una vez que se realicen las cosecha les serán entregadas.

En el subsistema indirecto corto el principal agente que interviene en el proceso de comercialización son los minoristas en centros poblados a los cuales el productor se ve obligado a entregar dadas las limitaciones para acceder al consumidor en forma directa, y porque los precios que consigue por su producto con los otros agentes es menor, así como por las exigencias de estos mercados. Esto significa que los pequeños productores agrícolas al momento de comercializar sus productos se desenvuelven en un mercado altamente competitivo y excluyente, las normas y reglas que se imponen para asegurar la calidad del producto favorecen a un modelo de desarrollo que privilegia la reproducción del dinero constituyéndose en la piedra angular de los procesos, productivos, comerciales y políticos (Novoa, 2013). 


\section{Volumen de ventas}

Los resultados de la investigación muestran un volumen total de ventas anual de $\$$ 736.173,6 USD, lo que representa un promedio de venta por feria de \$184.043,4 USD.

Tabla 4. Valores de los productos comercializados por los productores - feriantes en el mes

\begin{tabular}{lcccccc}
\hline Tipo producto & $\begin{array}{c}\text { Valor } \\
\text { percibido }\end{array}$ & $\%$ & $\begin{array}{c}\text { Valor no } \\
\text { vendido }\end{array}$ & $\%$ & $\begin{array}{c}\text { Valor } \\
\text { esperado }\end{array}$ & $\%$ \\
\hline Natural & $\$ 11.012$ & 87,7 & $\$ 1.551$ & 12,3 & $\$ 12.563$ & 100 \\
Semiprocesado & $\$ 1.614$ & 86,3 & $\$ 257$ & 13,8 & $\$ 1.871$ & 100 \\
Procesado & $\$ 2.711$ & 84,4 & $\$ 499$ & 15,6 & $\$ 3.211$ & 100 \\
\hline
\end{tabular}

Fuente: Elaboración propia a partir de los datos recolectados.

Puede decirse que existe un aumento de las ventas en las ferias solidarias de Imbabura en relación con años anteriores, lo cual se corresponde con lo alcanzado en la ciudad de lbarra en el estudio realizado.

Los productores han venido adaptando su sistema productivo a las necesidades de los consumidores, es decir, ampliando la diversificación de sus fincas (ofertando mayor diversidad de productos) y mejorando la planificación de siembra para garantizar la oferta de productos a lo largo del año. Otro factor favorable es la construcción de una relación de conocimiento mutuo productor - consumidor, que incentiva al consumidor a comprar ahora más alimentos que en años anteriores en esos espacios (Murillo \& Lacroix, pp.111-112).

Estos resultados permiten responder a la interrogante ¿es posible reproducir la Unidad Doméstica base de la Agricultura Familiar? ¿Por qué es tan importante dentro del estudio considerar esta pregunta? La teoría económica campesina menciona que la unidad doméstica de producción tiene como objetivo la reproducción y la subsistencia. En el trabajo usan mano de obra familiar, intercambian fuerza de trabajo entre parientes y amigos. Producen para el autoconsumo y el autoabasto y el excedente es para el mercado. No emplean capital, no trabajan bajo la lógica de optimización y maximización capitalista (Córdova, 2018). 
Ventura (2018) refiere en su trabajo Representaciones sobre el trabajo y la política social: un estudio de caso sobre el rol de los recursos económicos en las estrategias de reproducción familiar que la unidad doméstica, en tanto organización multifacética, contiene tres dimensiones: la material, expresada en bienes y servicios concretos que garantizan la supervivencia de sus miembros, la afectiva, que comprende los lazos familiares y las relaciones sociales; y la simbólica, ligada al capital social, a las ideologías y valores propios de cada clase. Es decir que mientras las familias se constituyen sólo a partir de lazos de parentesco, las unidades domésticas refieren además a un ámbito donde se crean y recrean relaciones de producción y condiciones reproducción, así como también se desarrollan relaciones de poder, autoridad, conflicto y solidaridad.

Tal como lo explican Ariza y Oliveira, las unidades domésticas:

Son también ámbitos primarios de convivencia en los que se establecen relaciones de cooperación, intercambio y negociación entre hombres y mujeres de diferentes generaciones. Las relaciones familiares son fundamentalmente de carácter asimétrico, jerárquico, porque siguen la distribución interna de poder que marcan dos ejes básicos de diferenciación social: el género y la generación (2003, p.22).

El entorno de la agricultura familiar campesina del Ecuador, como un ámbito de la economía solidaria, evidencia tres aristas fundamentales (Zárate, 2015):

a) la reproducción de la vida a partir de sistemas de relaciones sociales y comunitarias que generan prácticas productivas y económicas que pueden definirse dentro de la economía solidaria. Se pondrá un especial énfasis en los principios agroecológicos de producción;

b) En relación al anterior, la agricultura familiar campesina se constituye como un espacio de emergencia, desarrollo y ejercicio de una acción colectiva rural y movimientos sociales vinculados a la realidad rural tanto desde un abordaje campesino como indígena, acción colectiva que ha impactado en el estado; 
c) Se trata finalmente, de un entorno - la ruralidad - que históricamente ha sido el objeto y destino de una serie políticas y programas estatales, que han procurado su transformación y que han impactado en la estructura del territorio.

Dentro del estudio dos variables permiten analizar el papel de la agricultura familiar como sustento de la reproducción de la vida, generadora de prácticas productivas y económicas. Por una parte, parentesco del personal de ayuda, y por la otra, pago por ayuda. Para la CEPAL (2016) la "agricultura familiar es una forma de clasificar la producción agrícola, forestal, pesquera, pastoril y acuícola gestionada y operada por una familia y que depende principalmente de la mano de obra familiar, incluyendo tanto a mujeres como a hombres" (p.15).

El parentesco del personal de ayuda está relacionado en un 74,4 por ciento con esposos e hijos, y por la otra, que solo el 16 por ciento del productor - feriante realiza pago por ayuda que va desde 0.80 USD hasta 10 USD.

\section{Parentesco personal de ayuda}

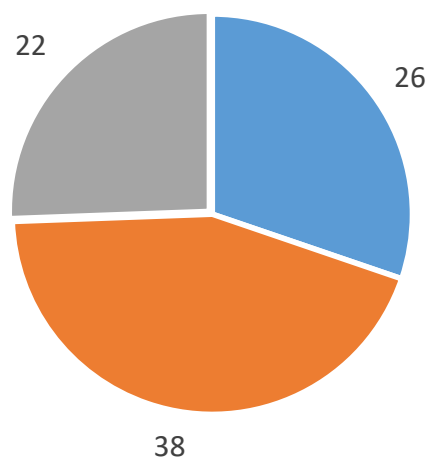

- \%

- Totales

Gráfico 3. Parentesco del personal de ayuda.

Fuente: Elaboración propia a partir de los datos recolectados. 


\section{Pago por ayuda}

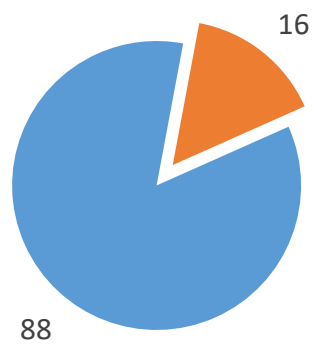

no pagan $\quad$ Si pagan

Gráfico 4. Pago por ayuda.

Fuente: Elaboración propia a partir de los datos recolectados.

Para Andino (2013) la mayor parte de mecanismos de apoyo previstos, tienden a promover más la integración de la economía popular y solidaria en el mercado (a través de apoyos a la generación de valor agregado, la industrialización, la compra pública y la exportación de sus productos), más que a fortalecer e incentivar mecanismos de reciprocidad y autoconsumo. Esto evidencia una desvalorización del potencial productivo, social y cultural de la agricultura familiar y la economía popular en general y más aún del potencial transformador de economías no mercantiles basadas y fundamentadas en las relaciones sociales y valores culturales de la gente. Se cree que la acción del Estado debe enfocarse en salvaguardar asistencialmente la supervivencia mínima de sujetos que considera no viables en sentido económico.

No obstante, este criterio es contrastado por Eguía y Ortale (2007) al desarrollar su investigación vinculada al concepto de Estrategias Familiares de Reproducción Social. Las dimensiones a partir de las cuales las autoras estudian las estrategias son:

- Estrategias laborales (acciones desarrolladas por los miembros de las unidades domésticas para obtener ingresos monetarios mediante el ingreso formal o informal al mercado de trabajo), que reside en la participación en las ferias solidarias como productores - feriantes;

- Participación en programas sociales, más vinculadas al papel del Estado en el desarrollo de políticas de asistencia social; 
- Acceso a redes informales de ayuda;

- Estrategias vinculadas con el proceso de salud, enfermedad y atención.

Existe también el elemento de identidad cultural que se ha constituido en un vehículo para la conformación de esta experiencia. Se trata de la vida comunitaria y la generación de lazos de parentesco que comprometan y acerquen a los miembros de una comunidad. Estos lazos se generan mediante la producción de dependencia mutua y la estimulación de la participación en iniciativas colectivas (Latouche, 2004; Narotsky, 2013).

\section{CONCLUSIONES}

Las ferias solidarias en la ciudad de lbarra se consideran circuitos cortos de comercialización, en base a la venta directa que se establece entre productores - feriantes y consumidores. Los por cientos alcanzados en la variable origen de los productos agroalimentarios se expresan a favor de un origen propio lo cual reduce al mínimo la intermediación, lo que permite una lógica pedagógica que contribuye a una mayor autonomía de los actores y, con ello, a una mayor sostenibilidad e integración social.

La unidad doméstica como núcleo de la agricultura familiar desarrolla estrategias cuyo sentido y fuerza intrínseca está dado por la reproducción de la vida de sus miembros, y cuya principal fuente es la efectivización de sus capacidades de trabajo, determinado como útil desde esa perspectiva y no desde la de la rentabilidad de un supuesto capital. Los valores alcanzados por la comercialización de sus productos en las ferias solidarias de la ciudad de lbarra constituyen una fuente directa de satisfactores

De forma general, las ferias solidarias en Ibarra deben comprenderse en el contexto de unas relaciones sociales de producción marcadas no solo por vínculos de tipo económico, sino también por relaciones étnico culturales. La dinámica desarrollada se ha desarrollado en función del mejoramiento de la calidad de vida de vida de los feriantes - productores. 


\section{REFERENCIAS BIBLIOGRÁFICAS}

Abreu, J. L. (2014). El Método de la Investigación Research Method. Daena: International Journal of Good Conscience, 9(3), 195-204.

Álvarez, J. A. Á., \& Maldonado, M. A. C. (2009). La dinámica social y el sistema político. Criterio Jurídico Garantista, vol. 1, no 1.

Andino, Verónica (2013). Políticas públicas para la economía social y solidaria Caso de estudio de Ecuador. Quito: RELIESS.

Ariza, M. y Oliveira, O., (2003) Acerca de las Familias y los Hogares: estructura y dinámica. Familia, trabajo y género. Un mundo de nuevas relaciones. Buenos Aires, Fondo de Cultura Económica-Unicef.

Bolívar, C. R. (2008). El enfoque multimétodo en la investigación social y educativa: una mirada desde el paradigma de la complejidad. Teré: revista de filosofía y sociopolítica de la educación, (8), 13-28.

Calle, D. y Zapata, A. (2013). Dinámicas de comercialización de la producción agropecuaria campesino indígena en la provincia de Imbabura. Dinámicas de comercialización para la agricultura familiar campesina: desafíos y alternativas en el escenario ecuatoriano. Quito, Ecuador, Agrónomos y Veterinarios sin Fronteras (AVSF), Sistema de Investigación sobre la Problemática Agraria en el Ecuador (SIPAE).

CEPAL, N. (2016). Encadenamientos productivos y circuitos cortos: innovaciones en esquemas de producción y comercialización para la agricultura familiar. Análisis de la experiencia internacional y latinoamericana.

Claveria, A. V., Díaz, S. C., \& Carrasco, D. M. (2018). El consejo de curso y la formación ciudadana: un espacio natural del profesor orientador. Revista de Orientación Educacional, 32(61), 79-94. 
Córdova-Ávalos, V. (2018). Factores que afectan la producción de cacao (Theobroma cacao L.) en el ejido Francisco I. Madero del Plan Chontalpa, Tabasco, México. Universidad y Ciencia Volumen 17. Número 34.

Driessnack, M., Sousa, V. y Costa, I. (2007). Revisión de los diseños de investigación relevantes para la enfermería: parte 3: métodos mixtos y múltiples. Revista LatinoAmericana de Enfermagem, 15(5), 179-182. Recuperado el 23 de noviembre del 2018. http://www.scielo.br/pdf/rlae/v15n5/es_v15n5a24.pdf

Eguía, A. y Ortale, S. (coordinadoras) (2007). Los significados de la pobreza. Editorial Biblos, Buenos Aires.

Gago, V., Cielo, C., \& Gachet, F. (2018). Presentación del dossier. Economía popular: entre la informalidad y la reproducción ampliada. Íconos: Revista de Ciencias Sociales, (62), 11-20.

Guevara, S., Arciniegas, M., \& Guerrero, W. (2017). Alternativas de desarrollo comunitario y ferias solidarias en Imbabura. Revista Publicando, 4(11 (2)), 545-569. Recuperado el 23 de noviembre de 2018. https://rmlconsultores.com/revista/index.php/crv/article/view/596

INEC, I. (2010). Instituto Nacional de Estadísticas y Censos. Recuperado el 23 de noviembre de 2018. http://www.ecuadorencifras.gob.ec/institucional/home.

Guridi, L., Pérez de Mendiguren, J.C. (2008). La Economía Social y Solidaria, las capacidades colectivas, y el desarrollo humano local. La economía social y solidaria como estrategia de desarrollo en el contexto de la integración regional latinoamericana1. Revista Foro, (66), 27-33.

Marsollier, R. G. (2018). Empleo Público y Desgaste Profesional. Un Análisis desde las Características de la Tarea. Revista Interamericana de Psicología Ocupacional, 35(2), 80-89. 
Mera, G. (2013). Análisis del desarrollo socioeconómico de los productores agrícolas en las ferias solidarias de la provincia de Imbabura, durante el periodo 2010 al 2012. Tesis de ingeniería, Universidad Técnica del Norte, Ibarra, Ecuador.

Murillo, R., \& Lacroix, P. (2014). Ferias solidarias en Ecuador: Alternativa para los pequeños productores locales y para un consumo sano y solidario en los territorios. Ferias y mercados de productores: hacia nuevas relaciones campo-ciudad. Recuperado el 1 de septiembre de 2018. https://tinyurl.com/y72xng7k

Narotsky, Susana (2013). Economías cotidianas, economías sociales, economías sostenibles. España. Icaria.

Novoa, B. (2013). Abastecimiento alimentario de los programas sociales a través del sistema de contratación pública del Ecuador. En V. Proaño y P. Lacroix (Ed.), Dinámicas de comercialización para la agricultura familiar campesina: desafíos y alternativas en el escenario ecuatoriano dinámicas de comercialización para la agricultura familiar campesina: desafíos y alternativas en el escenario ecuatoriano (pp. 99-138). Quito, Ecuador: Sistema de Investigación sobre la Problemática Agraria en el Ecuador (SIPAE).

Lacroix, P., \& Cheng, G. (2014). Ferias y mercados de productores: Hacia nuevas relaciones campo-ciudad. Lima: CEPES, AVSF. Recuperado el 27 de diciembre de 2018.

file:///C:/Users/hp/Documents/Instaladores/libro regional andino ferias mercados productores avsf cepes 2014.pdf

Latouche, S. (2004). Survivre au développement. Éditions mille et une nuits, les petits libres № 55, Paris.

Pérez, Z. P. (2011). Los diseños de método mixto en la investigación en educación: Una experiencia concreta. Revista electrónica educare, 15(1), 15-29. 
Pierri, M. C. Q. M., \& VALENTE, A. (2010). A feira livre como canal de comercialização de produtos da agricultura familiar. In CONGRESSO DA SOBER (Vol. 49). Recuperado $\begin{array}{lllll}\text { el } & 27 & \text { de diciembre } & \end{array}$ file://C:/Users/hp/Documents/Instaladores/A\%20FEIRA\%20LIVRE\%20COMO\%20 CANAL\%20DE\%20COMERCIALIZAÇÃO\%20DE\%20PRODUTOS\%20DA\%20AG RICULTURA\%20FAMILIAR\%20.pdf

Pinto, G. (2016). Las ferias solidarias en la provincia de Imbabura: desafios y ventajas del comercio justo. Tesis para obtener el título de Ingeniera en Economía mención Finanzas. UTN. Ibarra. Ecuador.

Vega, M. L., \& Ramírez, D. C. (2018). Startup en las redes sociales. Revista ESPACIOS, 39(27).

Velarde Cruz, E. (2013). Análisis de vulnerabilidades a nivel municipal-perfil territorial canton San Miguel de Ibarra. Recuperado el 23 de noviembre de 2018. http://dspace.cedia.org.ec/bitstream/123456789/848/1/Perfil\%20territorial\%20IBAR $\underline{\text { RA.pdf }}$

Ventura, M. V. (2018). Representaciones sobre el trabajo y la política social: un estudio de caso sobre el rol de los recursos económicos en las estrategias de reproducción familiar. Master's thesis, Buenos Aires: FLACSO. Sede Académica Argentina.

Zárate Baca, É. A. (2015). Ferias agroecológicas en la sierra norte de Pichincha entre la institucionalización de la economía solidaria y la acción colectiva rural. Master's thesis, Quito: FLACSO Sede Ecuador. 